\title{
A Simulation-Based Empathy Enhancement Program for Non-Medical Care Providers of Older Adults: A Mixed-Methods Study
}

\author{
Areum $\operatorname{Han}^{1}$ and Tae Hui Kim ${ }^{2} \bowtie$ \\ 1'Department of Occupational Therapy, University of Alabama at Birmingham, Birmingham, Alabama, USA \\ ${ }^{2}$ Department of Psychiatry, Yonsei University Wonju College of Medicine, Wonju, Republic of Korea
}

\begin{abstract}
Objective Studies using simulation-based programs for empathy enhancement have been conducted mostly for health profession students and medical care providers in Western countries. No empirical research has been conducted for non-medical care providers of older adults in community settings in Asian countries. The purposes of this mixed-methods study were: to explore experiences and perceived usability of non-medical care providers of older adults in a simulation-based empathy enhancement program; and to examine if the program is effective in improving empathy and relevant outcomes.

Methods 104 non-medical care providers of older adults in South Korea participated in a simulation-based empathy enhancement program in 2018. Data were collected using self-reported questionnaires for effectiveness testing, a program evaluation questionnaire, and individual interviews and analyzed using statistical tests and thematic analysis.

Results Care providers showed higher levels of empathy and lower levels of stress and burnout after the program participation ( $<<0.05$ ). Qualitative findings supported the improved attitude and care strategies, increased empathy towards older adults, preparing for their own aging, and restoration of emotional stability through the participation in the program.

Conclusion This study suggests that the simulation-based program is useful in promoting empathic responses of non-medical care providers working with older adults. Psychiatry Investig 2021;18(2):132-139
\end{abstract}

Key Words Compassion fatigue, Empathy, Health personnel, Quasi-experimental studies, Simulation training.

\section{INTRODUCTION}

The need for care providers of older adults has increased with the growth of the aging population worldwide including older adults living alone. ${ }^{1}$ Care providers play important roles in identifying living conditions and welfare needs in older adults and providing education and emotional support to older adults in the community. ${ }^{2}$ Care providers' empathy is regarded as one of the critical competencies to understand situations of older adults better and to take empathetic actions. ${ }^{3}$ Empathy is defined as 'an understanding of experiences, con-

Received: July 30, 2020 Revised: November 2, 2020

Accepted: November 14, 2020

$\triangle$ Correspondence: Tae Hui Kim, MD

Department of Psychiatry, Yonsei University Wonju College of Medicine, 20 Ilsan-ro, Wonju 26426, Republic of Korea

Tel: +82-33-741-0534, E-mail: gooddr@yonsei.ac.kr

(a) This is an Open Access article distributed under the terms of the Creative Commons Attribution Non-Commercial License (https://creativecommons.org/licenses/by$\mathrm{nc} / 4.0$ ) which permits unrestricted non-commercial use, distribution, and reproduction in any medium, provided the original work is properly cited. cerns, and perspectives of clients, the ability to communicate this understanding, and the intention to help' (p.74). ${ }^{4}$ Studies have found that care providers' empathy is positively associated with better health and well-being in clients and negatively associated with care providers' burnout and stress. ${ }^{5-7}$

Experiencing aging-related challenges and debriefing in a simulation-based empathy enhancement program can be useful for care providers to understand perspectives of older adults better possibly leading to improvement in the quality of care and positive outcomes in both care providers and older adults. ${ }^{8,9}$ Because care providers have not personally experienced challenges in older adults, such as challenges in performing daily activities and emotions resulting from challenges in older adults, it is not easy for care providers to understand and empathize with older adults fully. Experience learning is learning through experience, involving a process of interpreting experience with personal thoughts and emotions and planning new actions based on the learned experience. ${ }^{10}$ Experiential learning using simulation has been used to enhance empathy 
and empathetic behaviors of health profession students and care providers. ${ }^{9,11}$ Also, literature supports the negative relationship between care providers' empathy and burnout/stress and empathy as a protective factor of burnout in care providers, suggesting the potential effects of empathy training in decreasing care providers' burnout and stress. ${ }^{7,12}$

Mixed results have been found in empirical studies of empathy training programs including simulation-based programs overall, and the characteristics of the empathy training programs, participants, and methodology used in the literature varied. ${ }^{9,13}$ A meta-analysis of 18 randomized controlled trials found that empathy training programs are effective in improving empathy levels overall with a medium effect. ${ }^{13}$ However, the majority of studies included in the meta-analysis had small sample sizes and the characteristics of the included studies varied. Also, previous studies using aging simulation-based programs have been conducted mostly for health profession students and medical care providers in Western countries. No empirical research has been conducted for non-medical care providers of older adults in community settings in Asian countries. Findings of previous studies and the gap in the literature indicate the need of further studies to better understand how a simulation-based empathy training program is experienced and perceived by non-medical care providers of older adults in community-settings in Asian countries and how the program affects empathy and relevant outcomes in these care providers.

The purposes of this mixed methods study, thus, were: to explore experiences of non-medical care providers of older adults in community-settings in a simulation-based empathy enhancement program; to examine care providers' perceived usability of the program by considering usefulness, satisfaction, and ease of learning; and to test if the program is effective in improving empathy and relevant outcomes.

\section{METHODS}

\section{Design}

The current study was part of a larger study that assessed the effects of two empathy enhancement programs on 209 care providers of older adults using a non-equivalent control group pretest-posttest design. The present paper focused on data of 104 care providers who participated in a simulationbased empathy enhancement program using both quantitative and qualitative approaches (i.e., phenomenology to explore experiences in the program and quantitative data analysis to measure pretest-posttest differences in outcomes).

\section{Recruitment of participants}

Participants were recruited by using convenience sampling from August to November, 2018, and data collection and program implementation were done between September and November, 2018. Recruitment was done from social/senior welfare centers in four urban areas and two rural areas in Gangwon province, South Korea. Potential participants were informed about the research, and those who expressed interests in study participation were contacted by the research team to go through the informed consent process. Participants were eligible for inclusion if they were: social workers who managed public social services for older adults or direct care workers who provided regular check-in services through home visits and telephone to older adults living alone in the community; having direct interactions with older adults at the time of recruitment; aged $\geq 18$ years; and cognitively and physically able to participate in the program. The study was conducted with Institutional Review Board (IRB) approval from the corresponding author's university, South Korea (protocol \# CR318026).

\section{The simulation-based empathy enhancement program}

The simulation-based empathy enhancement program was modified from the previous pilot study of a dementia simulation program, ${ }^{14}$ originally developed by the AGE-u-cate Training Institute in USA (Dementia Live ${ }^{\mathrm{TM}}$ program). The original program was developed based on person-centered dementia care, need-driven, dementia-compromised behavior model, and experiential learning model. ${ }^{10,15,16}$ The program was modified by changing focus from dementia to aging and adding a brief breathing meditation session. The program aimed to help care providers enter the world of older adults by experiencing similar feelings and behaviors of older adults in the experience room and reflecting on their experiences from the perspectives of older adults in the empowerment session. In addition, the program aimed to help care providers cultivate mindfulness by considering the evidence supporting mindfulness as a potential method for reducing stress while increasing empathy. ${ }^{17}$ Even a single-session brief mindfulness training was found to be beneficial in increasing mindfulness and improving clinical skills. ${ }^{18,19}$ The entire simulation program took about 50 minutes including: a preparation session; an experience room session that required the completion of daily life tasks with limited sensory and cognitive functions; an empowerment session to debrief their performance, reactions, and behaviors in the experience room and to discuss why and what changes in care strategies for older adults were needed; and a brief mindfulness practice session.

\section{Data collection}

Quantitative data to measure the programs' effectiveness were collected before and two weeks after the program par- 
ticipation through the use of self-report questionnaires, with reliability and validity corroborated in Korean samples. Questionnaires included the Korean versions of: 1) the Empathy Quotient-Short form (EQ-Short), assessing a single-factor structure of empathy $\left.{ }^{20} ; 2\right)$ the Health Professional Version of the Jefferson Scale of Empathy (JSE-HP), assessing the selfperceived empathic behaviors of health professionals in the context of health care with three subscales: perspective taking, compassionate care, and standing in the patient's shoes ${ }^{21} ; 3$ ) the Professional Quality of Life Scale 5 (ProQoL5), assessing professionals perceived compassion satisfaction and compassion fatigue (burnout and secondary traumatic stress) $\left.{ }^{22} ; 4\right)$ the Caring Efficacy Scale (CES), assessing self-efficacy related to the caring attitudes and behaviors of professionals in the relationship with clients ${ }^{23}$; and 5) the Psychosocial Well-being Indexshort form (PWI-Short form), measuring the psychosocial stress level. ${ }^{24}$

A program evaluation questionnaire was developed to measure the program's usability by considering usefulness, satisfaction, and ease of learning. ${ }^{25}$ The questionnaire included 10 closed-ended questions on a scale of 1 (strongly disagree) to 5 (strongly agree) and 3 open-ended questions. Three openended questions included: 1) write three words that describe the characteristics of the program you participated in; 2) describe the advantages of the program and benefits of the program participation on yourself if any; and 3) describe the disadvantages of the program and any suggestion for the program improvement. Care providers completed the questionnaire two weeks following their participation in the program.

Individual interviews with 104 care providers who participated in the simulation-based program were done using a semi-structured interview guide developed based on recommendations. ${ }^{26}$ Each interview began with a general question asking about the program experience and further open-ended questions to explore the perceived impact of the program participation on their perspectives and caring for older adults.

\section{Data analysis}

Quantitative data analysis, including closed-ended questions of the program evaluation questionnaire, was performed using IBM SPSS Statistics 25.0. Internal consistency of 10 closedended questions in the program evaluation questionnaire was measured with Cronbach's alpha. Cronbach's alpha was 0.937 that are considered of excellent internal consistency. ${ }^{27}$ Descriptive statistics were used to summarize care providers' characteristics. The Shapiro-Wilk test and Kolmogorov-Smirnov test were used to test the normality of data. A paired t-test and Wilcoxon Signed rank test were used to determine the significance of differences in dependent variables for within-group analysis of the pretest and posttest results. Effect size (Cohen's $\mathrm{d}$ ) was calculated to measure the sizes of pretest-posttest differences, and the following intervals were applied for interpretations: $0.2-0.5$ representing a small effect size; $0.5-0.8$ representing a medium effect size; and 0.8 and higher representing a large effect size. ${ }^{28,29}$ The Bonferroni correction was used to avoid the increased risk of type I error. The level of significance was set at $\mathrm{p}<0.05$ for all tests.

For qualitative data analysis, ATLAS.ti 8 software (https:// atlasti.com) was used to analyze responses on open-ended questions of the program evaluation questionnaire and interview data by using word frequency queries and coding. For open-ended question 1 of the questionnaire, a word frequency query was run to identify which words were most often used by care providers to describe the characteristics of the program and program experience. For open-ended questions 2 and 3 of the questionnaire, we used the auto coding function to start coding under each question, created and coded at nodes, and reviewed and reorganized nodes. For analysis of the interview data, Braun and Clarke's six analytic steps for doing thematic analysis was used. ${ }^{30}$ The six analytic steps include: familiarizing with data (step 1) by reading and rereading the transcripts and making notes initial ideas; generating initial codes (step 2) by using line-by-line coding/open coding to organize data into small chunks of meaning; searching for themes (step 3) by selecting similar codes and grouping them into potential themes; reviewing themes (step 4) by checking if the preliminary themes work relative to the coded extracts and modifying themes as needed; defining and naming themes (step 5) by refining each theme and generating clear definitions and names for each theme; and producing the report (step 6) by selecting vivid extract examples and producing a report of the analysis. Strategies such as member checking and audit trail were used to increase the trustworthiness of the qualitative data analysis process. ${ }^{31}$ Interpretations and derived meanings against the transcript text were constantly checked to verify that the themes remained reflective of the transcript text, in an effort to increase analytical rigor.

\section{RESULTS}

\section{Characteristics of participants}

Table 1 describes general characteristics of 104 care providers who participated in the simulation-based empathy enhancement program. The even number of social workers and direct care workers participated in the simulation-based program. The majority of participants were female similar to the high proportion of females in South Korean social workers and direct care workers for older adults in the community. ${ }^{32}$ About $70 \%$ had religions and $64 \%$ obtained social work licenses. 
Table 1. General characteristics of participants $(\mathrm{N}=104)$

\begin{tabular}{lc}
\hline \multicolumn{1}{c}{ Variables } & $\mathrm{N}(\%)$ or $\mathrm{M} \pm \mathrm{SD}$ \\
\hline Age & $45.86 \pm 10.78$ \\
Gender & $18(17.3)$ \\
Male & $86(82.7)$ \\
Female & \\
Current job & $52(50.0)$ \\
Social worker & $52(50.0)$ \\
Direct care worker & \\
Education level & $2(1.9)$ \\
Middle school & $24(23.1)$ \\
High school & $63(60.6)$ \\
College & $15(14.4)$ \\
Graduate and above & \\
Marital status & $77(74.0)$ \\
Married & $17(16.3)$ \\
Never married & $5(4.8)$ \\
Widowed & $5(4.8)$ \\
Divorced & \\
Religion & \\
No religion & $14(13.5)$ \\
Protestantism & $27(23.1)$ \\
Catholicism &
\end{tabular}

M: mean, SD: standard deviation

\section{Effects of programs on care providers}

Table 2 shows differences in pretest and posttest scores in 104 care providers who participated in the simulation-based empathy enhancement program. Statistically significant pretest-posttest differences in outcome measures were found. After the intervention, the care providers had significantly higher levels of empathy measured by JSE-HP, including total scale ( $\mathrm{p}=0.033, \mathrm{~d}=0.428$ ) and Standing in the Patient's Shoe subscale $(\mathrm{p}=0.010, \mathrm{~d}=0.520)$. In addition, the care providers showed significantly lower levels of compassion fatigue, measured by Secondary Traumatic Stress scale of ProQoL5 ( $\mathrm{p}=0.022, \mathrm{~d}=$ 0.184 ) and Burnout scale of ProQoL5 ( $\mathrm{p}=0.015, \mathrm{~d}=0.490)$ respectively, after the intervention.

\section{Findings of the program evaluation questionnaire}

Table 3 shows scores in the closed-ended questions of the program evaluation questionnaire with the number and percent of care providers who agreed or strongly agreed with the statement among 104 care providers. A total of $95 \%$ of care providers in the simulation program reported that the program helped understanding of older adults and thinking from the perspectives of older adults. About 90 percent of care providers reported that the program was helpful to themselves overall and they recommend the program to care providers of older adults.

Word frequency query results on open-ended question 1 showed that care providers described the program most frequently as the following words: 'empathy' (14.7\%), 'understanding' (13.5\%), 'firsthand experience' (9.0\%), 'consideration' $(4.5 \%)$, 'hearing problem' (3.5\%), 'frustrated' $(3.2 \%)$, 'embarrassed' (2.6\%), 'confused' (2.6\%), and so on. The most often reported responses regarding the program's advantages and benefits included: 'increased empathy toward older adults/ being in their shoes' (29.8\%); 'firsthand experience helped understanding' (26.0\%); 'overall understanding about older adults' (24.0\%); 'improved care strategies' (13.5\%); 'understanding about difficulties in daily life due to changes in physical functions' (12.5\%); 'understanding about sensory and perceptual changes in older adults' (11.5\%); and 'an opportunity to think about my attitudes and care strategies toward older adults' (7.7\%); 'understanding about emotions/psychological issues in older adults' (7.7\%); and 'understanding about behaviors exhibited by older adults' (6.7\%). The most frequently reported responses as the disadvantages of the program and any suggestion for the program improvement involved: 'a need to cover more comprehensive and various aspects of aging simulation experience' (23.1\%); 'the program was too short, wanting to spend a longer time for the simulation experience' (11.5\%); 'a need to have more realistic simulation setting and equipment/ tools' (8.7\%); and 'want an individual session as well as a group session' (2.9\%).

\section{Findings of the individual interviews}

Five key themes emerged relating to care workers' perspectives on the simulation-based program experience.

\section{Theme 1. Improved attitude and care strategies}

Improved care strategies by participating in the simulation program were reported by care providers, including improved communication strategies and relationship-centered care approaches. Improved communication strategies included: 'to talk briefly, clearly, and slowly; to use non-verbal communication such as eye contact, gestures, touch, and tone of voice; to repeat; to check if older adults understood what the care pro- 
Table 2. Pretest-posttest comparisons in outcome measures $(\mathrm{N}=104)$

\begin{tabular}{|c|c|c|c|c|c|}
\hline Variables & Pretest $(\mathrm{M} \pm \mathrm{SD})$ & Posttest $(\mathrm{M} \pm \mathrm{SD})$ & t or Z & $\mathrm{p}$ & Cohen's d \\
\hline EQ-short & $10.39 \pm 3.23$ & $10.65 \pm 3.56$ & $-1.167^{\ddagger}$ & 0.243 & 0.230 \\
\hline \multicolumn{6}{|l|}{ JSE-HP } \\
\hline Total & $104.87 \pm 10.07$ & $106.20 \pm 11.37$ & $-2.133^{\ddagger}$ & $0.033^{*}$ & 0.428 \\
\hline PT & $58.28 \pm 5.39$ & $58.64 \pm 5.96$ & $-1.147^{\ddagger}$ & 0.251 & 0.226 \\
\hline $\mathrm{CC}$ & $35.93 \pm 5.31$ & $36.62 \pm 5.22$ & $-2.018^{\ddagger}$ & 0.044 & 0.404 \\
\hline SS & $8.37 \pm 2.46$ & $9.11 \pm 2.67$ & $-2.566^{\ddagger}$ & $0.010^{*}$ & 0.520 \\
\hline \multicolumn{6}{|l|}{ ProQoL5 } \\
\hline CS & $39.28 \pm 4.78$ & $39.21 \pm 4.92$ & $-0.285^{\ddagger}$ & 0.775 & 0.056 \\
\hline STS (-) & $27.00 \pm 4.60$ & $26.10 \pm 5.16$ & $2.329^{\dagger}$ & $0.022^{*}$ & 0.184 \\
\hline B (-) & $23.98 \pm 4.38$ & $23.17 \pm 4.42$ & $-2.426^{\ddagger}$ & $0.015^{*}$ & 0.490 \\
\hline CES & $116.39 \pm 12.52$ & $116.67 \pm 12.49$ & $-0.387^{\dagger}$ & 0.699 & 0.022 \\
\hline PWI-SF (-) & $16.85 \pm 6.47$ & $16.76 \pm 8.64$ & $-0.660^{\ddagger}$ & 0.509 & 0.130 \\
\hline
\end{tabular}

A minus sign in parentheses indicates that a decline in each variable means positive outcomes. ${ }^{*}$ statistically significant at $\mathrm{p}<0.05$, ${ }^{+}$assessed by

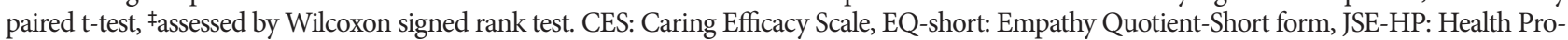
fessional Version of the Jefferson Scale of Empathy, JSE-HP-CC: compassionate care subscale of JSE-HP, JSE-HP-PT: perspective taking subscale of JSE-HP, JSE-HP-SS: standing in the patient's shoe subscale of JSE-HP, M: mean, ProQoL5: Professional Quality of Life Scale 5, ProQoL5-B: burnout subscale of ProQoL5, ProQoL5-CS: compassion satisfaction subscale of ProQoL5, ProQoL5-STS: secondary traumatic stress subscale of ProQoL5, PWI-SF: Psychosocial Well-being Index-Short form, SD: standard deviation

Table 3. Findings of the program evaluation questionnaire $(\mathrm{N}=104)$

\begin{tabular}{|c|c|c|}
\hline Questions & $\mathrm{M} \pm \mathrm{SD}$ & $\mathrm{N}(\%)$ \\
\hline Total scores & $4.20 \pm 0.54$ & \\
\hline Q1. The content was easy to understand. & $4.07 \pm 0.80$ & $84(81)$ \\
\hline Q2. The way the program was delivered was easy to understand. & $4.13 \pm 0.71$ & $88(85)$ \\
\hline Q3. The program helped me understand older adults. & $4.42 \pm 0.65$ & $99(95)$ \\
\hline Q4. The program helped me think from perspectives of older adults. & $4.52 \pm 0.62$ & $99(95)$ \\
\hline Q5. Overall, the program was helpful to me. & $4.23 \pm 0.66$ & $95(91)$ \\
\hline Q6. Overall, I was satisfied with the program. & $4.12 \pm 0.74$ & $88(85)$ \\
\hline Q7. I could apply what I learned from the program to my real life. & $3.95 \pm 0.76$ & $82(79)$ \\
\hline Q8. The program is useful in educating care workers of older adults. & $4.20 \pm 0.67$ & $91(88)$ \\
\hline Q9. I recommend the program to colleagues at my work. & $4.15 \pm 0.71$ & $89(86)$ \\
\hline Q10. I recommend the program to care workers of older adults. & $4.26 \pm 0.70$ & $93(90)$ \\
\hline
\end{tabular}

$\mathrm{N}$ (\%) means the number (\%) who agreed or strongly agreed with the statement among 104 care providers. M: mean, SD: standard deviation

vider said correctly; and to listen to what older adults want to say more.' Care providers' awareness in the importance of how to communicate with older adults were increased by directly experiencing failed communication when given the tasks to complete before entering the simulation room and its consequences such as not being able to complete tasks, and feeling frustrated with low self-esteem. Care providers who participated in the simulation program started using more relationship-centered care approaches rather than task-oriented care approaches by realizing the importance of relationships and understanding affect and emotion of older adults better. Also, care providers started using giving more time to older adults more often than before by understanding impacts of aging on completing daily tasks and consequences of such impacts on emotions of older adults in everyday life better. Care providers' improved care strategies affected older adults positively based on the reports of care providers who interacted with older adults for about two weeks after participating in the program: 'Older adults brightened/felt good/were satisfied by feeling understood'; 'Communication with older adults was enhanced'; and 'Older adults responded positively to a request'.

\section{Theme 2. Increased empathy towards older adults and understanding}

Care providers stated that the simulation-based program helped them have increased understanding of concerns and 
perspectives of older adults by standing in the shoes of older adults through the simulation situation. Such simulation experience helped care providers better understand how older adults would feel in their daily lives and behaviors of older adults the care providers could not understand before. Participation in the simulation program encouraged care providers to 'pay more attention to the challenges and dangers of everyday life of older adults' with increased understanding of consequences of aging. In particular, the program allowed care providers to have emotion-focused experiences by feeling confused, embarrassed, frustrated, helpless, and annoyed in the context of impaired sensory functions during the simulation situation. The program experience promoted care providers to 'be more willing to help older adults' by considering not only physical conditions of older adults but also emotional consequences of aging. For example, a social worker related her feelings of frustration and embarrassment in the simulation experience to feelings of low self-esteem that older adults would have in daily lives. The social worker realized the importance of considering varied aspects of aging, leading to being more patient and putting more effort in understanding older adults.

\section{Theme 3. Thinking about and preparing for their own aging and parents' aging}

Participation in the simulation program promoted a care provider to 'think about my life in old age' and have 'increased awareness in preparing for my aging and taking good care of my health.' Experiencing varied sensory, perceptive, cognitive, and emotional changes resulting from aging influenced care providers to have 'the will to live the present more meaningfully and healthy' In addition to increased awareness in their own aging, 'better acknowledging and understanding of their parents' aging' occurred from the simulation experience. The simulation experience facilitated a care provider to determine to 'be good for my parents and pay more attention to my parents' aging.'

\section{Theme 4. Restoration of emotional stability}

Participation in the simulation program was regarded as the experience that reduced stress at work and increased job satisfaction. It was regarded as the experience of regaining their emotional stability. Care providers reported such benefits resulted from better understanding about aging, realization of their improved care approaches, mindfulness, and realization of the value of their jobs. For example, a direct care worker 'felt ease after accepting that the older adult was not able to hear or understand what I said well or what I talked to the older adult was too long' rather than thinking that 'the older adult pretend not to know' after participating in the program.

\section{Theme 5. Overall experience in the program and suggestions}

Care providers recognized the simulation program as 'a great experience that was helpful to my job and others', 'a new interesting experience', and 'a firsthand experience better than lectures.' The following areas were recognized as the most helpful things by the care providers: 'improved empathy towards older adults and increased understanding of older adults; understanding the importance of communication and improved communication strategies; a will to take good care of my health for healthy aging; self-examination about my care strategies and attitudes; a mind of living the present meaningfully and thankfully; and paying more attention to older adults in needs.' Care providers made comments on the program and suggestions for improvement. These feedbacks included: 'providing the program to more people; providing more regular, sustained educational opportunities with emotional support like this program to care providers; the need and importance of the firsthand experience like this program; adding varied scenarios to apply varied aspects of aging experience to the simulation program; making the program longer because the current version is too short; and having more realistic setting.'

\section{DISCUSSION}

The current study examined the effectiveness and perceived usability of a simulation-based empathy enhancement program on 104 non-medical care providers of older adults in the community and explored care providers' experiences in the program. Significant improvements were found in empathy and compassion fatigue (i.e., stress and burnout) at posttest. Literature supports that empathy is negatively related with burnout and stress, and improving empathy is emphasized to protect work-related stress and burnout in health professions. ${ }^{33}$ It should be noted why improvements in empathy were found when measured by JSE-HP but not by EQ-short. JSE-HP was developed to assess empathy of care providers with focus more on cognitive empathy, or understanding another person's experiences, concerns, and perspectives. ${ }^{34}$ On the other hand, EQ-short was not developed to measure care providers' empathy by having questions not relevant to care settings. ${ }^{20} \mathrm{EQ}-$ short also does not differentiate cognitive aspects of empathy from affective aspects (i.e., sharing another person's emotion), believing that cognitive and affective aspects of empathy cooccur. ${ }^{20}$ No significant change in EQ-short might be because the simulation-based program in the present study enhanced cognitive empathy more than affective empathy and the EQshort could not capture improvements in cognitive empathy relevant to care settings. Significant changes in the overall JSEHP score and Standing in the Patient's Shoe subscale measur- 
ing cognitive empathy indicate that the simulation-based empathy program may help care providers better understand experiences and perspectives of older adults in particular. Cognitive empathy has been found to be a significant protector to burnout and stress of care providers. ${ }^{33}$ This explains why the simulation-based empathy enhancement program showed significant improvements in not only cognitive empathy but also burnout and stress to some extent.

Findings of the program evaluation questionnaire in the current study support usefulness perceived by care providers. Perceived usefulness in simulation-based programs have been supported in the literature to some extent. For example, a virtual reality intervention using a dementia simulation movie was regarded as useful in $85 \%$ of informal caregivers of people with dementia. ${ }^{35}$ A total of $76 \%$ of the caregivers reported that they changed their approach to caregiving and $61 \%$ stated that the intervention increased their understanding of dementia. ${ }^{35}$ In the present study, $95 \%$ of care providers in the simulation program reported that the program helped understanding of older adults and thinking from the perspectives of older adults. A total of $79 \%$ of care providers reported that they could apply what they learned from the program to their real lives. These findings of the current study showed similar but higher percentage of usefulness perceived by care providers compared to caregivers of people with dementia in the previous study. ${ }^{35}$

Five key themes emerged relating to care providers' perspectives in the simulation-based program experience, including improved attitude and care strategies, increased empathy towards older adults, preparing for their own aging, restoration of emotional stability, and overall experience in the program and suggestions. These qualitative findings complement findings of the program evaluation questionnaire and improvements in outcome measures to some extent. Improved care approaches (e.g., verbal and nonverbal communication strategies) in addition to improved empathy were reported by the care providers in the simulation program. In a follow-up study of the authors, older adults living alone $(n=100)$ expressed significantly higher levels of satisfaction and improved emotions when the older adults were interviewed about health, emotional status, and lifestyle using the same protocols by care providers who received the simulation-based program compared to those who did not receive the simulation-based program. ${ }^{36}$ Such findings support findings of the present study, indicating the simulation-based program increased care providers' ability to communicate empathic understanding toward older adults.

Suggestions made by the care providers of the simulationbased program should be considered for improvement of the program. Further studies are needed to apply varied aspects of aging experience to the simulation program with a longer period of time for care providers to experience in a more realistic setting. Some care providers commented on applying more typical physical conditions of older adults such as pain and muscle loss on the body to the simulation program. Also, scenarios should be developed further to simulate more realistic experiences of various older adults including older adults living alone. One possible method is to use virtual reality technology for more realistic simulation of older adults who may experience loneliness and anxiety of living alone as an older adult.

Due to several limitations of the current study, findings should be interpreted with caution. A further randomized controlled trial study is needed to examine the program's effectiveness compared to a control group with minimal bias and confounding factors. Also, it is unknown how long the benefits of the one-time empathy enhancement program would last. Further studies should consider having a follow-up evaluation to measure its long-term effect on outcomes. In addition, providing an additional session for behavioral skills training (e.g., instruction, modeling, practice, and feedback) after participating in the simulation program may help care providers to apply more practically useful skills with higher motivation to help and enhanced empathy. ${ }^{13}$ Benefits on care providers also might be maximized if the simulation program is combined with a lecture-based education program based on person-centered care approaches. ${ }^{37}$ The findings in this study contribute to the lack of evidence in simulation-based empathy enhancement programs for non-medical care providers of older adults in community-settings.

\section{Acknowledgments}

This research was supported by a grant of the Korea Health Technology R\&D Project through the Korea Health Industry Development Institute (KHIDI), funded by the Ministry of Health \& Welfare, Republic of Korea (grant number: HI18C1207).

\section{Conflicts of Interest}

The authors have no potential conflicts of interest to disclose.

\section{Author Contributions}

Conceptualization: Areum Han, Tae Hui Kim. Methodology: Areum Han, Tae Hui Kim. Software: Areum Han. Validation: Tae Hui Kim. Formal analysis: Areum Han. Investigation: Areum Han, Tae Hui Kim. Data curation: Areum Han. Writing — original draft: Areum Han. Writing—review\& editing: Tae Hui Kim.

\section{ORCID iDs}

Areum Han

Tae Hui Kim

https://orcid.org/0000-0003-1369-9052

https://orcid.org/0000-0003-0133-5227

\section{REFERENCES}

1. Reher D, Requena M. Living alone in later life: a global perspective. Popul Dev Rev 2018;44:427-454. 
2. Lyu I. A study of life satisfaction level concerning the life management assistant dispatch project for the elderly living alone. Korean J 21 Century Soc Welfare 2008;5:159-180.

3. Gerdes KE, Segal E. Importance of empathy for social work practice: integrating new science. Soc Work 2011;56:141-148.

4. Hojat M. Empathy in Health Professions Education and Patient Care. New York, NY: Springer International; 2016.

5. Derksen F, Bensing J, Lagro-Janssen A. Effectiveness of empathy in general practice: a systematic review. Br J Gen Pract 2013;63:e76-e84.

6. Kelm Z, Womer J, Walter JK, Feudtner C. Interventions to cultivate physician empathy: a systematic review. BMC Med Educ 2014;14:219.

7. Lee PT, Loh J, Sng G, Tung J, Yeo KK. Empathy and burnout: a study on residents from a Singapore institution. Singapore Med J 2018;59:50-54.

8. Anderson K, Bird M, MacPherson S, Blair A. How do staff influence the quality of long-term dementia care and the lives of residents? A systematic review of the evidence. Int Psychogeriatr 2016;28:1263-1281.

9. Bearman M, Palermo C, Allen LM, Williams B. Learning empathy through simulation: a systematic literature review. Simul Healthc 2015; 10:308-319.

10. Kolb DA. Experiential Learning: Experience As the Source of Learning and Development. Englewood Cliffs, NJ: Prentice Hall; 1984.

11. Ter Beest $H$, van Bemmel M, Adriaansen M. Nursing student as patient: experiential learning in a hospital simulation to improve empathy of nursing students. Scand J Caring Sci 2018;32:1390-1397.

12. Thirioux B, Birault F, Jaafari N. Empathy is a protective factor of burnout in physicians: new neuro-phenomenological hypotheses regarding empathy and sympathy in care relationship. Front Psychol 2016;7:763.

13. Teding van Berkhout E, Malouff JM. The efficacy of empathy training: a meta-analysis of randomized controlled trials. J Couns Psychol 2016; 63:32-41.

14. Han A, Kim TH, Hong H. Experiences of caregivers of people with dementia in a Korean dementia simulation program. Dementia 2019: 1471301218823453.

15. Algase DL, Beck C, Kolanowski A, Whall A, Berent S, Richards K, et al. Need-driven dementia-compromised behavior: an alternative view of disruptive behavior. Am J Alzheimer Dis 1996;11:10-19.

16. Brooker D. What is person-centred care in dementia? Rev Clin Gerontol 2003;13:215-222.

17. Bohecker L, Doughty Horn EA. Increasing students' empathy and counseling self-efficacy through a mindfulness experiential small group. J Spec Group Work 2016;41:312-333.

18. Mahmood L, Hopthrow T, Randsley de Moura G. A moment of mindfulness: computer-mediated mindfulness practice increases state mindfulness. PLoS One 2016;11:e0153923.

19. Gockel A, Burton D, James S, Bryer E. Introducing mindfulness as a selfcare and clinical training strategy for beginning social work students. Mindfulness 2013;4:343-353.

20. Yeo J. Validation of the Korean Version of the Empathy Quotient-Short form. J Kor Acad Industr Coop Soc 2012;13:5356-5363.
21. Ryu H, Bang K. A validation study of the Korean version of the Jefferson Empathy Scale for Health Professionals for Korean nurses. J Korean Acad Nurs 2016;46:207-214.

22. Joo H, Cho Y, Ahn H. Validation of the Korean version of Professional Quality of Life Scale 5 (K-ProQOL 5) for mental health professional. Cognit Behav Ther Korea 2016;16:269-298.

23. Kim Y. A study on the validity of Caring Efficacy Scale for social workers. J Korean Soc Welfare Admin 2017;19:103-129.

24. Chang S. Standardization of Collection and Measurement of Health Statistics Data. Seoul: Korean Society for Preventive Medicine; 2000.

25. Lund AM. Measuring usability with the USE questionnaire. Usabil Interface 2001;8:3-6.

26. Smith J, Flowers P, Larkin M. Interpretative Phenomenological Analysis: Theory, Method, and Research. London, England: Sage Publications; 2009.

27. Tavakol M, Dennick R. Making Sense of Cronbach's Alpha. Int J Med Educ 2011;2:53-55.

28. Cohen J. Statistical Power Analysis for the Behavioral Sciences (2nd Ed.). Hillsdale, NJ: Lawrence Erlbaum Associates; 1988.

29. Morris SB, DeShon RP. Combining effect size estimates in meta-analysis with repeated measures and independent-groups designs. Psychol Methods 2002;7:105-125.

30. Braun V, Clarke V. Using thematic analysis in psychology. Qual Res 2006;3:77-101.

31. Nowell LS, Norris JM, White DE, Moules NJ. Thematic analysis: striving to meet the trustworthiness criteria. Int J Qual Methods 2017;16: 1609406917733847.

32. Korea Association of Social Workers. 2018 Statistical Yearbook of Social Workers. Available at: https://www.043w.or.kr/DATA/wml/ e9976234-7142-48e1-9725-dbc14cdd47b8.pdf. Accessed July 5, 2020.

33. Wagaman MA, Geiger JM, Shockley C, Segal EA. The role of empathy in burnout, compassion satisfaction, and secondary traumatic stress among social workers. Soc Work 2015;60:201-209.

34. Hojat M, DeSantis J, Shannon SC, Mortensen LH, Speicher MR, Bragan L, et al. The Jefferson Scale of Empathy: a nationwide study of measurement properties, underlying components, latent variable structure, and national norms in medical students. Adv Health Sci Educ Theory Pract 2018;23:899-920.

35. Jütten LH, Mark RE, Sitskoorn MM. Can the mixed virtual reality simulator into dementia enhance empathy and understanding and decrease burden in informal dementia caregivers? Dement Geriatr Cogn Dis Extra 2018;8:453-466.

36. Park S, Kim TH, Um TR, Lee K, Jung J, Hong M, et al. The effects of simulation-based empathy enhancement program for care of the elderly on counselling. Innovation in Aging 2019;3(Suppl 1):S920-S921.

37. Han A, Kim TH, Hong H. A factorial randomized controlled trial to examine separate and combined effects of a simulation-based empathy enhancement program and a lecture-based education program on family caregivers of people with dementia. Aging Ment Health 2020:1-11. 